\title{
VLBI Observations of Flares in RS CVn Binary Systems
}

\author{
Corrado Trigilio ${ }^{1}$, Grazia Umana ${ }^{1}$, Victor Migenes ${ }^{2}$ \\ 1 Istituto di Radioastronomia del C.N.R., C.P. 169 Noto, Italy \\ 2 C.S.I.R.O., ANU Campus, GPO Box 3023, Canberra 2601, Australia
}

\section{Introduction}

RS Cvn systems have been studied very extensively at radio wavelengths and a large fraction of them have been found to be strong radio sources, with $6 \mathrm{~cm}$ radio luminosities in the range between $10^{15}$ and $10^{18} \mathrm{erg} \mathrm{s}^{-1}$. The radio flux density is highly variable and it usually shows two different regimes: active periods, characterized by a continuous strong flaring which can last for several days, and quiescent periods, during which the flux density goes down to a few mJy. These characteristics indicate the non-thermal nature of the radio emission which is driven by magnetic activity of these systems, seen in other spectral regions. In this scenario, the radio flux arises from the interaction between the magnetic field of one or both components with mildly relativistic particles, i.e. gyrosynchrotron emission.

Due to the erratic nature of radio flares in RS CVn binaries, most VLBI observations have been performed during quiescent periods. Only the 1983 UX Ari observation (Mutel et al. 1985), carried out during an intense outburst, allowed the first, and still the unique, hybrid map of a RS CVn binary, which revealed a core-halo morphology of the radio source.

It is still not clear if the flare events are localized in very compact regions of the corona of one component or if the entire binary system is involved. VLBI measurements in both active and quiescent periods are extremely important to determine the characteristic dimensions and the structure of the radio source.

\section{Single dish monitoring program}

In 1991 we have started a $6 \mathrm{~cm}$, single dish monitoring of active binary systems, using the $32 \mathrm{~m}$ telescope at the Noto VLBI station of the Istituto di Radioastronomia of C.N.R. The main aim of these systematic total power observations is to activate ad-hoc VLBI observations once a flaring period is detected. Four binary systems have been selected as targets for the monitoring program: HR 1099, UX Ari, HR 5110 and Algol. Those system are the only ones for which information of flaring events with flux densities higher then $250 \mathrm{mJy}$ are available. 
One of the most interesting results of this program comes from the monitoring of HR 1099 (V711 Tau). This is a non-eclipsing RS CVn binary system consisting of a $\mathrm{K} 1$ subgiant and a G5 main sequence star, with an orbital period of 2.8 days and a separation between the two components of about $11.5 R_{\odot}$.

The single dish measurements show that there are long active periods during which strong radio flares occur one after the other and the flux density never reaches its quiescent value. On the other hand, the quiescent phase can last for several weeks.

\section{VLBI observations}

\subsection{The 1991 July 26 observation}

In July 1991 HR 1099 experienced a series of strong radio flares, one of which reached a flux density of $\approx 800 \mathrm{mJy}$. Just after the main flare event, which occurred on July 25, an ad hoc VLBI observation run was carried out (Trigilio et al. 1993), giving for the first time the opportunity to measure the brightness temperature trend during a flare decay in a RS CVn binary. The observations were made, on July 26 , at $6 \mathrm{~cm}$ in standard VLBI Mark II mode, with a $2 \mathrm{MHz}$ bandwidth. Three telescopes (Noto, Jodrell Bank and Cambridge) were involved. From the single dish measurements performed just before and after the VLBI run, it was clear that we were following the decay of the flare; the flux went from $\approx 660 \mathrm{mJy}$ to $\approx 360 \mathrm{mJy}$ in about five hours. Due to the source variability we split the 3 hours observing time into 6 different, 30 min scans. We have separately fitted the data from each scan to a two-dimensional Gaussian brightness distribution and derived the source angular dimension and the corresponding linear size, assuming a distance of $35 \mathrm{pc}$. The angular size of the radio source was found to be about 4 mas, which corresponds to a linear size almost twice as large as the overall binary size. The dimensions and the observed flux density allowed us to determine the brightness temperature, using the relation:

$$
T_{\mathrm{B}}=1.97 \times 10^{6} \text { Flux }(\mathrm{mJy}) \frac{\lambda^{2}(\mathrm{~cm})}{\theta_{\text {mas }}^{2}}
$$

Because the source size did not change during our observation, apart for a possible expansion between the first and the second scan, we can exclude any contribution of structural changes of the radio source to the flare decay. We can, therefore, conclude that the observed flux density variation is due entirely to a decay of emitting particles energy.

\subsection{The 1992 April 29 and 30 observations}

The April 29 and 30 VLBI observations were performed at the frequency of $5 \mathrm{GHz}$ in MK II recording mode, with a bandwidth of $2 \mathrm{MHz}$. Three European antennas started the observations, joined by five VLBA antennas, with two hours of simultaneous observations; the VLBA ended the observations at $02 \mathrm{UT}$ of 30 
1992 April 29

1992 April 30

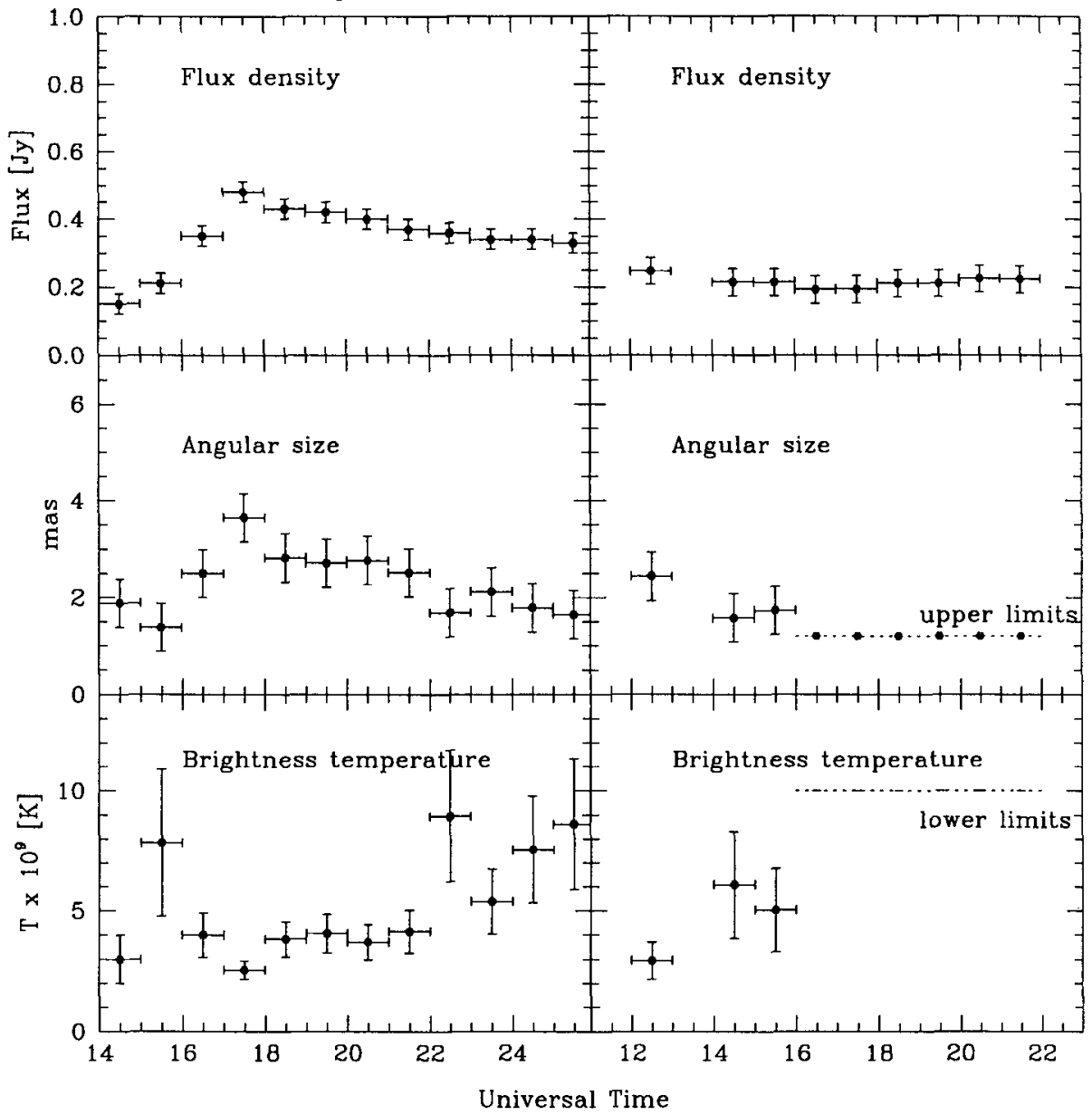

Fig. 1. Radio light curve, source size and brightness temperature evolution for the 29 and 30 April 1992 VLBI observations.

April (for the first day) and at 22 UT of 30 April (for the second day). The visibility function was analyzed as for the July 91 observations. The flux density radio light curve, together with the angular size and brightness temperature evolution are shown in Fig 1.

On April 29, we observed a complete flare: the source reached a maximum flux density of about $500 \mathrm{mJy}$ in about 3 hours, then a very slowly decay, lasting more than 8 hours, occurred. The source was very compact at the beginning, being comparable with the size of the $\mathrm{K}$ star; an expansion followed which involved the whole binary system. During the decay, the size of the source was almost constant, showing a decrease at the end. The brightness temperature was high 
at the beginning of the flare, decreased during the expansion, and at the end, it increased drastically.

The decay of this flare is much longer than the one in July 1991. A possible interpretation can be given. The brightness temperature beaviour indicates that an energy release occurred at the beginning, when the expansion started, then loss mechanisms operated in the corona, as the decay seems to indicate. In the second part of the observations a new ejection of energetic particles occurred. The long flux decay seems to be sustained by a new flare.

During the April 30 observations, the activity level was still high, showing, however, a different trend. The flux density was almost constant (about 250 mJy) during the whole observation, lasting more than 10 hours. The source size decreased, beeing comparable with the entire binary system at the beginning of the observations. The source was not spatially resolved in the second part, so only an upper limit of 1.2 mas can be estimated. The brightness temperature was increasing, showing a lower limit of $10^{10} \mathrm{~K}$ at the end.

These data seem to indicate that in the first part the decay of a previous flare has been observed, with a source as big as the whole system (halo) and a low brightness temperature. Then multiple flares, or a continuous particle ejection occurred in a very compact layer (core).

\section{Conclusions}

The single dish monitoring program gives the possibility to organize VLBI observations during flares. In fact, due to the erratic nature of the radio emission in RS CVn binary systems, it is impossible to foresee when an active period will start. VLBI observations give the possibility to follow the size and the brightness temperature evolution during these events, and this provides inportant constraints to any attempt to model stellar coronae.

From the three VLBI observations of HR 1099 a qualitative picture of the radio flare dynamic can be drawn. A flare first develops in a compact region (comparable with the stellar size), then the source expands, involving the whole system. The relativistic electrons lose energy via radiative decay, collisions and other possible mechanisms, and the typical decay time is about 5-6 hours. A flare is often a non unique event, and sometimes new flares occur during the decay, providing energetic electrons. Longer decay times can be observed in this case.

\section{References}

Mutel R.L., Lestrade J.F., Preston R.A., Phillips R.B., 1985, ApJ 289, 262

Trigilio C., Umana G., Migenes V., 1993, MNRAS 260, 903 